\title{
MAPPING THE GALACTIC CENTER REGION WITH GRASP
}

\author{
Ph.Durouchoux ${ }^{1}$; G.Bignami'; A.Dean ${ }^{3}$; N.Lund ${ }^{4}$; B.McBreen ${ }^{5}$; \\ V.Schonfelder ${ }^{6}$; B.Swanenburg ${ }^{7}$; G.Vedrenne ${ }^{8}$; C.Wirkler ${ }^{9}$.
}

- 1 CEN Saclay BP No2 F 91, Gif sur Yvette France - 2 IFCTR/CNR Via Bassini 15,20133 Milano, Italy. -3 Physics Dept.univ.ofSouthampton, UK. - ${ }^{4}$ Danish Space Research Inst. Copenhagen, Denmark - 5 Physics Dept.University College, Dublin 4, Ireland - ${ }^{6}$ Max Planck Institute for Physics and Astrophysics D-8046 Garching, FRG. ${ }^{7}$ ROL, Leiden, The Netherlands. ${ }^{8}$ CESR, 9 av du colonel Roche 31029 Toulouse, France. $\therefore$ ESAESTEC Postbus 299, 22400 AG Noordwijk, The Netherlands.

\section{ABSTRACT}

The GRASP mission (Gamma-ray Astronomy with Spectroscopy and Positioning ) is currently under study as an ESA space astronomy mission to be launched in the mid 90's. GRASP is designed as a high quality spectral imager $(E / \Delta E \approx 500$ at $1 \mathrm{Mev})$ with positioning to the arc minute level within a large field of view $\left(\approx 7^{\circ}\right)$ which operates over a wide spectral range ( $30 \mathrm{Kev}-100 \mathrm{Mev}$ ) with a $3 \sigma$ sensitivity of typically 10 mcrab or better over the entire operational range within an observational period of $\approx 10^{5}$ seconds. In this paper, we will mainly discuss the capability of the instrument with respect to the study of both point source and diffuse source measurements of the galactic center region.

\section{INTRODUCTION}

The GRASP telescope, which may be the first high resolution spectral imager to operate in the gamma-ray region in space will have the following features:

- a wide operational bandwidth (4 Kev to $100 \mathrm{Mev}$ ) which for the first time, links $X$-ray and $\gamma$ ray astronomy. 
- high resolution spectrometry over the range $30 \mathrm{Kev}$ to $10 \mathrm{Mev}$ $(E / \Delta E \approx 500$ at $1 \mathrm{Mev})$

- accurate source positioning (typically $\approx 1$ arc $\min$ ) within a field of view of $\approx 50$ square degrees.

- high sensitivity for both extended and point sources (typically 10 mcrab at $3 \sigma$ in $10^{5} \mathrm{~s}$ )

These wide ranging goals are achieved by the use of combination of a coded aperture mask and a position sensitive detector plane associated with a mosaic of high energy resolution detectors. The gamma-ray detector plane consists of an assembly of CsI(TI) scintallators and germanium solid state detectors arranged into an overall position sensitive array.

\section{IDESCBIPTION OE THE GRASP TELLSCOPE}

The principal characteristics of the GRASP instrument (fig 1) are:

- a mosaic of 19 stacks of hyperpure $G e$ detectors for low energy photon detection (20Kev-10 Mev). Each stack consists of 4 planar type detectors (each $5.4 \mathrm{~cm}$ diameter, $1.5 \mathrm{~cm}$ thick).

The overall spectral resolution is of the order of $2 \mathrm{Kev}$ at $1 \mathrm{Mev}$ and the geometric area $\approx 400 \mathrm{~cm}^{2}$. This configuration, advantageous in terms of the rejection of $\beta^{ \pm}$decay background, has a low total background level and is resistant to radiation damage.

- an array of $3 \mathrm{D}$ position sensitive CsI(TI) scintillators capable of both locating and measuring the energy deposited by particle interactions throughout the detector volume, corresponding to a sensitive area of $\approx 2400 \mathrm{~cm}^{2}$.

- a Stirling cycle cooler system associated with a passive radiator for the solid state spectrometer make a long mission possible (> 3 years)

- an active shield for the solid state spectrometer consisting of an array of Csl scintillators (mean thickness:12 cm).

- an hexagonal URA mask located about 4 meters "above" the detector plane gives a point source location capability of the order of the one arc minute. 
The narrow line and broad line sensitivities of the germanium and CsI detectors for $10^{5} \mathrm{~s}$. and $10^{6} \mathrm{~s}$. observation periods are shown in fig 2 . and 3.

Typically these $3 \sigma$ sensitivities are of the order of a few times $10^{-6}$ photons. $\mathrm{cm}^{-2} \cdot \mathrm{s}^{-1}$ at $1 \mathrm{Mev}$ for narrow lines and a few times $10^{-7}$ photons. $\mathrm{cm}^{-2} \cdot \mathrm{s}^{-1}$ at $10 \mathrm{Mev}$ for broad lines.

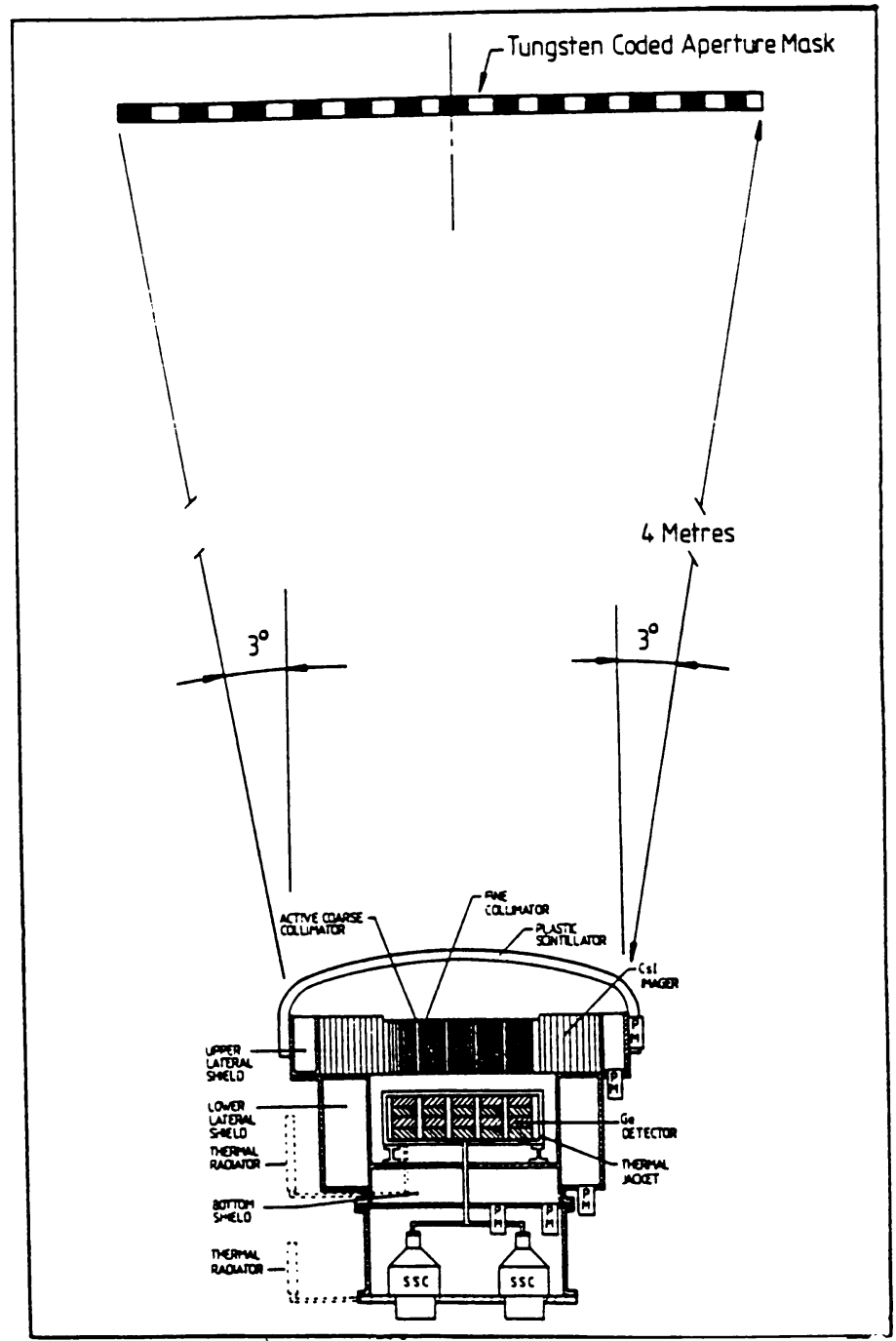

Fig 1 Schematic diagram of the GRASP telescope: 
The continuum sensitivity for GRASP is presented in fig 4 (where a 10 mcrab spectrum is shown as reference)

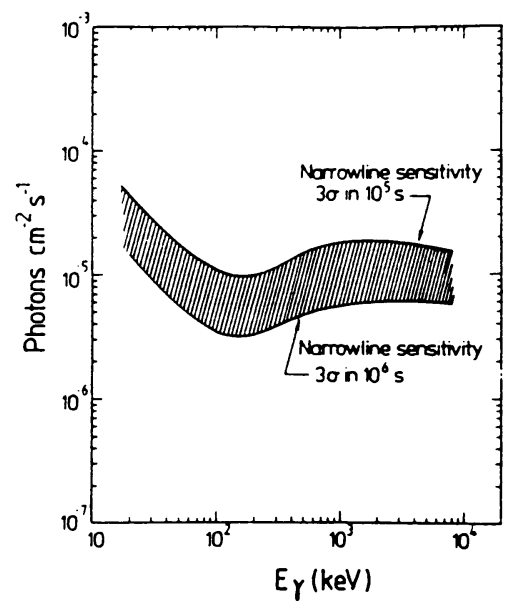

Fig 2: Narrow line sensitivity of the GRASP telescope ( $\mathrm{Ge}$ detectors)

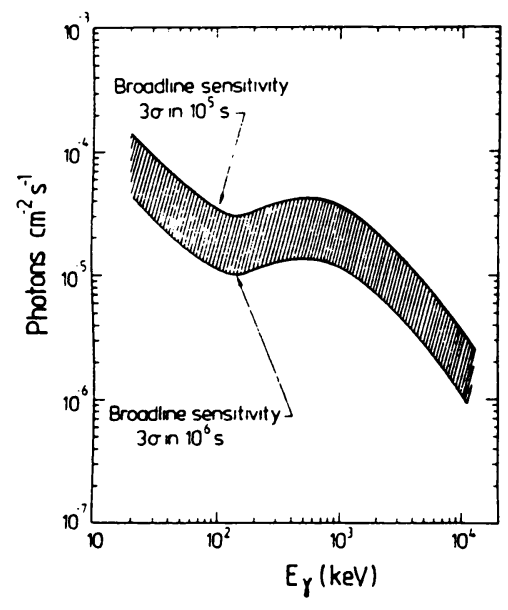

Fig 3: Broadline sensitivity of the GRASP telescope (CsI(TI) detectors

\section{ILASTROPHYSICAL OBJECTIVES}

\section{1- Extragalactic objects}

The study of active galaxies is to be one of the major features of the mission.

The unambiguous identification of a large number of actives galaxies will lead directly to the compilation of a gamma-ray luminosity function for these objects in the region of the spectrum where their liminosity is at a maximum.

Futhermore the detailed study of red-shifted electron-positron annihilation lines from these distant sources has fundamental cosmological implications.

Precise measurements of both the line and continuum spectra will provide a revealing probe of the physics in the vicinity of the compact objects associated with extragalactic nuclei. 
The study of explosive nucleosynthesis in local supernovae $(<10$ Mpc) will be also possible.

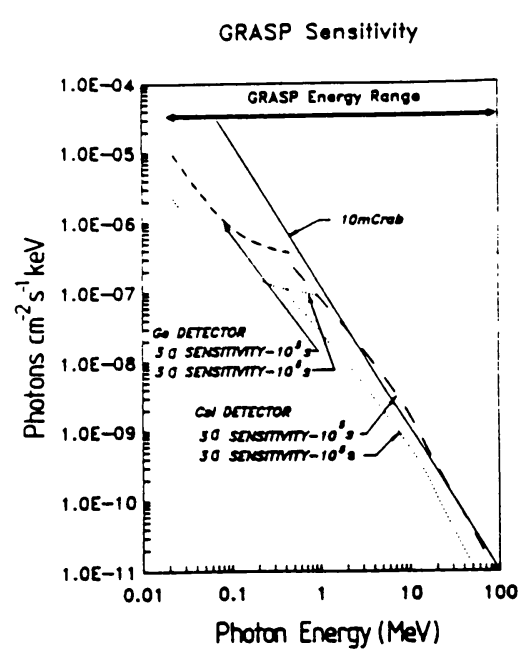

Fig 4: Continuum sensitivity of the GRASP telescope

\section{2- Galactic objects}

In the context of our galaxy, GRASP

will discover new gamma-ray sources, map extended objects, locate point sources precisely ( $\approx 1$ arc $\min$ ) analyse their emission spectra with high resolution and study the variability of a wide variety of spectral objects, with special em-phasis on the galactic center. A picture of the distribution of the recent products nucleosynthesis in the galaxy will be derived by mapping key emission lines $\mathrm{Al}^{26}$ and $\theta^{-}+\theta^{+}$annihilation.

This has direct bearing on the stu-dy of stellar nucleosynthesis e.g. in the Red Giant and Wolf-Rayet phases.

Also galactic novae are exciting targets for high resolution spectroscopy studies, as they are potential explosive nucleosynthesis sites. Finally, $\gamma$ rays coming from the interaction of cosmic rays with the interstellar medium are interesting goals for observations.

\section{MISSION AND SCENARIO}

This mission is under phase A study at the European Space Agency for a final selection which will take place in November 1988. This phase $A$ is accompanied by industrial studies for a space platform which might satisfy the GRASP requirements, allowing about $10^{3}$ pointings in the sky, lasting $10^{5}$ to $10^{6} \mathrm{~s}$.

This would maximise the scientific output of the mission, and offer a significant opportunity of a benificial fallout on the wider astronomical community through an Associate Observer Program which was investigate during the GRASP Workshop held at the Observatoire de PARIS-MEUDON on May 31st-June 1st \& 2nd 1988. 